\title{
Factores relacionados con la seguridad y la calidad en la atención del paciente pediátrico hospitalizado
}

\section{Factors related to safety and quality of attention in hospitalized pediatric patients}

\section{Fatores relacionados à segurança e qualidade no atendimento pediátrico hospitalizado}

\author{
J. Barrientos Sánchez ${ }^{\mathrm{a}{ }^{*}}$, M. Hernández Zavala ${ }^{\mathrm{b} 1}$, R.A. Zárate Grajales ${ }^{\mathrm{c} 2}$ \\ ORCID: \\ a $0000-0002-5794-0066$ \\ b 0000-0002-4013-3209 \\ c 0000-0002-9264-8490 \\ ${ }^{1}$ Instituto Nacional de Pediatría, Ciudad de México, México \\ ${ }^{2}$ Escuela Nacional de Enfermería y Obstetricia, Ciudad de México, México \\ Trabajo derivado del proyecto multicéntrico PAPIIT-UNAM IN304414 \\ Recibido: 2 marzo 2018 \\ Aceptado: 14 octubre 2018
}

\section{Resumen}

Introducción: La atención proporcionada a los usuarios de los sistemas de salud puede derivar con relativa frecuencia en un evento adverso (EA), debido a factores intrínsecos, extrínsecos o del sistema.

Objetivo: Analizar los factores relacionados con la calidad y la seguridad de los pacientes pediátricos hospitalizados, a través de los reportes de eventos adversos.

Métodos: Estudio derivado de un proyecto multicéntrico, transversal, prospectivo. Unidad de observación: todos los eventos adversos reportados en niños hospitalizados de 0 a 18 años, en un Instituto Nacional de Salud de la Ciudad de México, de junio de 2014 a julio de 2015. Se utilizó el instrumento SYREC para el reporte. Análisis estadístico descriptivo y comparación de variables mediante Chi cuadrada y prueba exacta de Fisher.

Resultados: Se registraron 173 eventos adversos, 55\% fueron del sexo masculino, mayor ocurrencia < 1 año (43\%), áreas críticas $60 \%$. Principales eventos: extubaciones no programadas (16\%), UPP (14\%), flebitis 
$(10 \%)$, lesiones $(9 \%)$, quemaduras $(8 \%)$ y medicamentos $(6 \%)$. Los factores del sistema se presentaron en 39\% (distracción, sobrecarga, falta de comunicación, falta de habilidad, supervisión). Factores extrínsecos 35\% (acceso a la monitorización, manejo terapéutico). Factores intrínsecos 26\% (condición clínica del paciente).

Conclusiones: La calidad y seguridad en la atención del paciente, se ve mermada principalmente por factores del sistema, esto debido a la amplia gama de funciones que debe realizar el profesional de enfermería, lo que conlleva a distracciones que diluyen el perfil de su rol y se apartan del objetivo principal que es el cuidado.

Palabras clave: Seguridad del paciente; calidad de la atención de salud; niño hospitalizado; México.

\section{Abstract}

Introduction: The attention provided to users of health systems can often end up in adverse events (AE) due to intrinsic or extrinsic system factors.

Objective: To analyze factors related to safety and quality of attention in hospitalized pediatric patients using an adverse-events reporting system.

Method: This is a study derived from a multicentric, transversal, and prospective project. Observation unit: All reported adverse events on hospitalized children aged $0-18$ at the National Institute of Health of the City of Mexico, from June 2014 to July 2015. The SYREC instrument was used for the report. A statistic descriptive analysis and variable comparison through Chi squared and Fisher exact test was performed. Results: 173 adverse events were registered, $55 \%$ of these on males, higher occurrence $<1$ year $(43 \%)$, in critical areas 60\%. The main events were: unprogrammed intubation (16\%), UPP (14\%), phlebitis $(10 \%)$, lesions $(9 \%)$, burns $(8 \%)$, medication-related $(6 \%)$. System factors $(39 \%)$ were related to distraction, overload, lack of communication, lack of ability, supervision. Extrinsic factors (35\%) were related to monitoring, therapeutic handling. Intrinsic factors (26\%) were related to the clinic condition of the patient.

Conclusions: Safety and quality of attention to hospitalized pediatric patients is often affected by diverse system factors, thus, nursing professionals should be aware of potential impacts to address them correspondingly.

Keywords: Patient safety; quality of health attention; child, hospitalized; Mexico.

\section{Resumo}

Introdução: A atenção dispensada aos usuários dos sistemas de saúde pode derivar com relativa frequência em um evento adverso (DA), devido a fatores intrínsecos, extrínsecos ou sistêmicos.

Objetivo: Analisar os fatores relacionados à qualidade e segurança de pacientes pediátricos hospitalizados, através dos relatos de eventos adversos.

Métodos: Estudo derivado de um projeto prospectivo, multicêntrico, transversal. Unidade de observação: todos os eventos adversos relatados em crianças hospitalizadas de 0 a 18 anos, em um Instituto Nacional de Saúde da Cidade do México, de junho de 2014 a julho de 2015. O instrumento SYREC foi usado para o relatório. Análise estatística descritiva e comparação de variáveis utilizando teste do qui-quadrado e teste exato de Fisher.

Resultados: foram registrados 173 eventos adversos, sendo 55\% do sexo masculino, maior ocorrência <1 ano (43\%), áreas críticas 60\%. Principais eventos: extubações não programadas (16\%), UPP (14\%), flebite $(10 \%)$, lesões $(9 \%)$, queimaduras $(8 \%)$ e medicamentos $(6 \%)$. Fatores do sistema foram apresentados em 39\% (distração, sobrecarga, falta de comunicação, falta de habilidade, supervisão). Fatores extrínsecos 35\% (acesso ao monitoramento, manejo terapêutico). Fatores intrínsecos 26\% (condição clínica do paciente). 
Conclusões: A qualidade e segurança da assistência ao paciente é principalmente afetada por fatores do sistema, devido à ampla gama de funções que o profissional de enfermagem deve realizar, o que leva a distrações que diluem o perfil de seu papel e eles partem do objetivo principal do cuidado.

Palavras chave: Segurança do paciente; qualidade dos cuidados de saúde; criança hospitalizada; México.

\section{Introducción}

La Organización Mundial de la Salud, en el año 2004, pone en marcha la Alianza Mundial por la Seguridad del Paciente, con el objetivo de prestar mayor atención a la seguridad de los mismos, fortalecer las bases científicas y fomentar la calidad de la atención en salud. A partir de entonces, diversas investigaciones se han realizado en torno a esta problemática ${ }^{1}$.

En España, el estudio ENEAS ${ }^{2}$, que ha tenido una importante repercusión, tanto dentro como fuera del país, por tratarse de uno de los estudios de mayor alcance realizados a nivel mundial, muestra que el 9.3\% de un total de 5, 624 pacientes, sufrieron un evento adverso durante la hospitalización.

La Comisión Nacional de Arbitraje Médico, como Centro Colaborador de la Organización Panamericana de Salud, desde el año 2009, cuenta con un Sistema de Registro Automatizado de Incidentes en Salud (SIRAIS), en el que contempla a instituciones hospitalarias públicas y privadas del territorio mexicano de segundo y tercer nivel de atención, el cual señala que a la fecha no existe obligación del reporte, por lo que hay subregistro con la consecuente pérdida de datos de los eventos suscitados ${ }^{3}$.

De esta manera, la mayoría de los reportes de eventos adversos (EA), registrados a nivel nacional e internacional, cuentan con un subregistro que oscila entre el 8 y el $26 \%$, la literatura refiere que sus principales causas derivan de la carga de trabajo, el sentimiento de culpa en el trabajador, la cultura institucional punitiva, entre otros ${ }^{4,5}$.

Desde hace décadas las alianzas bilaterales entre hospitales destinadas a mejorar la seguridad del paciente y la calidad de la atención, han servido para el intercambio técnico entre el personal sanitario. Tales alianzas ofrecen un canal de aprendizaje bidireccional sobre seguridad del paciente y la posibilidad de encontrar, conjuntamente, soluciones en unos sistemas de salud mundiales que cambian con rapidez $z^{6,7}$.

Entre el $20 \%$ y el $40 \%$ de todo el gasto en salud, se despilfarra a causa de una atención de calidad deficiente, los estudios sobre seguridad muestran que en algunos países se pierden hasta US\$19 000 millones por año, a causa de la prolongación de la estancia en el hospital, los litigios, las infecciones intrahospitalarias, la discapacidad, la pérdida de productividad y los gastos médicos. Así pues, las razones económicas para mejorar la seguridad del paciente son poderosas ${ }^{8}$.

El EA se debe a una serie de sucesos ${ }^{9}$ que en ningún caso son responsabilidad de un solo individuo, ya que no se debe olvidar que cada actividad del proceso de atención en salud tiene por sí mismo un riesgo ${ }^{10,11}$, por lo que, para este estudio se tomó la clasificación utilizada en el instrumento SYREC, desarrollado en España y utilizado en otros países de Europa y Australia, adaptado en Colombia y México, el cual abarca: factores intrínsecos, extrínsecos y del sistema ${ }^{12}$.

Existen pocos estudios sobre EA en pediatría. La mayoría describen lo que sucede en medicación o bien extrapola a los niños lo que sucede en los adultos, aun cuando existen diferencias sustanciales entre ambas poblaciones (epidemiológicas, demográficas, de desarrollo y tipo de cuidado requerido). Son más frecuentes los EA en pediatría en niños hospitalizados que en otros escenarios de salud, debido principalmente a las condiciones de susceptibilidad y la intensidad de las actividades que se desarrollan en este ámbito ${ }^{10,13}$.

En México, la Red de unidades de investigación en enfermería ENEO, instituciones e institutos nacionales de salud (de la cual forma parte este Instituto), inició un proyecto donde se analiza esta temática en diversas instituciones ${ }^{14}$, y a partir de dicho estudio se derivan estos resultados referentes a población pediátrica, donde el objetivo fue: analizar factores relacionados con la calidad y seguridad de los pacientes a través de los EA ocurridos en las áreas de hospitalización pediátrica. 


\section{Métodos}

Se trata de un estudio, no experimental, analítico, transversal y prospectivo, el lugar de aplicación fueron los diferentes servicios de hospitalización y áreas críticas de la institución. La unidad de observación fueron los eventos adversos ocurridos en los niños hospitalizados de junio 2014 a julio de 2015, reportados por el personal de enfermería de cualquier turno.

Para recolectar los datos sobre los eventos adversos ocurridos, se utilizó el instrumento SYREC, aplicado en países de Europa y Australia, adaptado en Colombia y México, que está dividido en cinco secciones: 1. Identificación del paciente; contiene datos de sexo, edad, diagnóstico, días de estancia del servicio, turno e índice enfermera/paciente.

2. Eventos adversos reportados por enfermería; contemplan las siguientes categorías y sus indicadores correspondientes.

- Relacionados con los medicamentos: errores en la prescripción, transcripción, dispensación, preparación, administración y monitorización, así como una doble verificación.

- Relacionados con la sangre y sus componentes: presencia de reacciones transfusionales, paciente equivocado o producto equivocado.

- Relacionados con la vía aérea y ventilación mecánica: obstrucción de la vía aérea, durante la introducción del tubo, extubación no programada, atelectasia, reintubación, broncoaspiración, desconexión accidental de la ventilación, decanulación, o barotrauma.

- Relacionados con los accesos vasculares, sondas, tubos, drenajes: catéter venoso periférico, catéter venoso central, dispositivo intracraneal, catéter arterial, drenaje torácico, catéter de diálisis, drenaje quirúrgico, sonda vesical, catéter arterial pulmonar, sonda nasogástrica.

- Relacionados con falla de los equipos: cama, monitor, desfibrilador, humidificador, marcapaso, bomba de infusión, respirador, alarmas.

- Relacionados con error diagnóstico: enfoque médico incorrecto, valoración de enfermería incorrecta, interpretación incorrecta de pruebas, disponibilidad de medios diagnósticos adecuados y pruebas complementarias adecuadas.

- Relacionados con pruebas diagnósticas: radiología, neurofisiología, laboratorio, endoscopía, patología y otros.

- Relacionados con el cuidado: caída, inmovilización, presencia de lesiones por presión, no aplicación de cuidados pautados.

- Relacionados con el procedimiento: retraso evitable, procedimiento inadecuado, preparación inadecuada antes del procedimiento, monitorización inadecuada, daño inadvertido en un órgano, sangrado.

- Relacionados con infección nosocomial: infección por neumonía asociada al ventilador, bacteriemia asociada a catéter, infección de tracto urinario asociado a sonda uretral u otros.

- Relacionados con complicaciones quirúrgicas: derivado de la anestesia, paciente incorrecto, sitio incorrecto, hemorragia asociada al procedimiento, dehiscencia de suturas, infección de la herida quirúrgica, otras.

3. Clasificación y gravedad del evento adverso. Sistema de clasificación a partir del incidente y su relación con la gravedad, el cual se clasifica en nueve categorías que van de la A, donde no llega a producirse algún daño puesto que se descubre y soluciona, hasta la categoría I, donde la consecuencia es la muerte del paciente.

4. Grado de evitabilidad del suceso. Sin duda evitable, posiblemente evitable, sin duda no evitable.

5. Factores presentes en la aparición de los eventos adversos. Se divide en tres categorías con sus respectivos indicadores.

- Factores intrínsecos. Condición clínica, factores sociales, mentales, psicológicos y relaciones interpersonales. 
- Factores extrínsecos. Corresponden al tratamiento y cuidado intrahospitalario que se brinda al paciente, a través de dispositivos y manejo terapéutico (tratamiento terapéutico invasivo o no invasivo y difícil manejo terapéutico).

- Factores del sistema. Individuales del trabajador implicado: aspectos físicos (salud, discapacidad, fatiga), psicológicos (distracción, preocupación, drogas, alcohol, falta de atención, distracción, preocupación, sobrecarga, aburrimiento). Del equipo y sociales (incongruencia del rol y liderazgo). Comunicación (verbal y escrita, comprensión de órdenes). Ligados a la tarea (guías, protocolos, procedimientos y políticas). Ayuda a la toma de decisiones (libros, revistas, personal experto). Formación y entrenamiento (falta de conocimiento, habilidades, experiencia y disposición). Supervisión (falta de supervisión de personal experto). Equipamiento y recursos (falta de conocimiento sobre el funcionamiento de aparatos, disponibilidad de manuales, funcionamiento incorrecto). Del trabajo (Diseño de áreas de trabajo, relación personal/paciente, carga de trabajo, personal temporal, descanso insuficiente, tareas ajenas, presión de tiempo y emergencias).

Los métodos que se utilizaron para la organización de la información fueron con estadística descriptiva. Para caracterizar la muestra, se emplearon frecuencias y porcentajes, además con las variables edad, días de estancia, clasificación de la gravedad del paciente, hora de ocurrencia e índice enfermera paciente se determinaron medias, medianas, moda, desviación estándar, máximos y mínimos. Para establecer la comparación entre factores extrínsecos, intrínsecos y del sistema con las variables de caracterización de los EA y del paciente, se realizaron pruebas no paramétricas de Chi cuadrada y prueba exacta de Fisher, se reportan los datos más relevantes.

De acuerdo al Reglamento de la Ley General en Materia de Investigación para la Salud, el presente estudio se cataloga como investigación sin riesgo, ya que no se realizó ninguna intervención o modificación intencionada en las variables fisiológicas, psicológicas y sociales de los individuos, debido a que la unidad de análisis son los Eventos Adversos.

\section{Resultados}

Se registraron 173 eventos adversos, de los cuales 95 (55\%) se presentaron en el sexo masculino, la edad promedio fue de 4 años \pm 5.4 , sin embargo la edad de mayor ocurrencia se encuentra en los de $0-1$ año, 74 (43\%). Ocurrieron con mayor frecuencia en áreas críticas 104 (60\%), y dentro de los primeros 10 días de estancia hospitalaria 93 (54\%). De acuerdo al índice enfermera paciente, los porcentajes mayores en áreas críticas fue de $1: 2$, en 79 (46\%), y hospitalización 1:4 en 30 (17\%). De acuerdo al turno, el nocturno reportó 79 (46\%), vespertino 59 (34\%), y el matutino 34 (19\%).

De acuerdo a los eventos adversos reportados por enfermería, se encontró lo siguiente:

- Los eventos relacionados con medicamentos se presentaron en un 7.5\% (13), principalmente hubo error en la administración (velocidad de infusión inadecuada, administración de medicamento no indicado, omisión de dosis, paciente incorrecto y sobredosis).

- Sangre y hemoderivados $2.3 \%$ (4), transfusión a paciente equivocado, y producto equivocado.

- Vía aérea $21.3 \%$ (37): mayormente extubación no programada y obstrucción de vía aérea.

- En cuanto a los equipos 0.6\% (1): falla de ventilador (respirador).

- Sondas y acceso vasculares 15.6\% (27), principalmente accesos periféricos, centrales y arteriales, seguido de salida de sonda transesofágica y salida de aguja de catéter puerto.

- Error diagnóstico 0.6\% (1): valoración incorrecta de enfermería.

- En lo que respecta al cuidado 40\% (69), fueron los que se presentaron en mayor frecuencia, principalmente lesiones por presión, flebitis, lesiones en general (brazalete de TA, fijación de dispositivos como sondas y catéteres, vendajes, dispositivos como electrodos), quemaduras (saturómetro, soluciones de base, medicamentos, lámpara de calor, escaldadura) y caídas. 
- Procedimientos 11.5\% (20); principalmente durante el proceso de aspiración de secreciones (sangrados, aporte de oxígeno, colocación inadecuada de dispositivos, no seguimiento adecuado de procedimientos).

- Complicaciones quirúrgicas 0.6\% (1); fijación quirúrgica de sonda transpilórica.

- Pruebas diagnósticas e IAAS (Infecciones asociadas a la atención sanitaria) 0\% cada una. Tabla 1.

Tabla 1. Tipo de Evento Adverso $(n=173)$

\begin{tabular}{lrr}
\hline & $\mathbf{f}$ & $\%$ \\
\hline Medicamentos & 13 & 7.5 \\
\hline Sangre y hemoderivados & 4 & 2.4 \\
\hline Vía aérea & 37 & 21.3 \\
\hline Equipos & 1 & 0.6 \\
\hline Accesos vasculares y sondas & 27 & 15.6 \\
\hline Error diagnóstico & 1 & 0.6 \\
\hline Pruebas diagnósticas & 0 & 0 \\
\hline Cuidado & 69 & 40.0 \\
\hline Procedimientos & 20 & 11.5 \\
\hline Infecciones & 0 & 0 \\
\hline Complicaciones quirúrgicas & 1 & 0.6 \\
\hline Total & 173 & 100 \\
\hline
\end{tabular}

Del personal involucrado con el evento adverso, 156 (90\%) corresponden al profesional de enfermería, y $17(10 \%)$ a personal médico.

De acuerdo a la clasificación de la gravedad, en 68 casos (39\%), el incidente contribuyó o causó daño temporal al paciente y precisó intervención; en otros 10 casos además del daño temporal, contribuyó a prolongar la hospitalización. Tabla 2.

Tabla 2. Clasificación de la gravedad $(n=173)$

\begin{tabular}{|c|c|c|c|}
\hline Categoría & Descripción & $\mathbf{f}$ & $\%$ \\
\hline A (Cuasifalla) & $\begin{array}{l}\text { Circunstancias o situaciones con capacidad de producir un incidente, pero que no } \\
\text { llegan a producirlo por descubrirse y solucionarse antes de llegar al paciente }\end{array}$ & 7 & 4 \\
\hline B (Evento adverso) & $\begin{array}{l}\text { El incidente alcanzó al paciente y no le causó daño, no } \\
\text { requirió monitorización ni intervención }\end{array}$ & 23 & 13 \\
\hline C (Evento adverso) & $\begin{array}{l}\text { El incidente alcanzó al paciente y no le causó daño, pero precisó monitorización } \\
\text { y/o intervención para comprobar que no había causado daño }\end{array}$ & 43 & 25 \\
\hline D (Evento adverso) & El incidente causó un daño imposible de determinar & 15 & 9 \\
\hline E (Evento adverso) & El incidente contribuyó o causó daño temporal al paciente y precisó intervención & 68 & 39 \\
\hline F (Evento adverso) & $\begin{array}{l}\text { El incidente contribuyó o causó daño temporal al } \\
\text { paciente o prolongó la hospitalización }\end{array}$ & 10 & 6 \\
\hline G (Evento centinela) & El incidente contribuyó o causó daño permanente al paciente & 2 & 1 \\
\hline H (Evento centinela) & $\begin{array}{l}\text { El incidente comprometió la vida del paciente y se } \\
\text { precisó intervención para mantener su vida }\end{array}$ & 5 & 3 \\
\hline I (Evento centinela) & El incidente contribuyó o causó la muerte del paciente & 0 & 0 \\
\hline Total & & 173 & 100 \\
\hline
\end{tabular}

En la tabla 3, se observa que el profesional de enfermería, considera que los eventos adversos pueden ser evitables hasta en $93 \%$ de los casos. 
Tabla 3. Evitabilidad del suceso $(\mathbf{n}=173)$

\begin{tabular}{lrr}
\hline Evitabilidad & f & \% \\
\hline Sin Duda Evitable & 117 & 68 \\
\hline Posiblemente Evitable & 44 & 25 \\
\hline Posiblemente Inevitable & 10 & 6 \\
\hline Sin Duda Inevitable & 2 & 1 \\
\hline Total & 173 & 100 \\
\hline
\end{tabular}

En total fueron reportados 417 factores intervinientes, de los cuales, los del sistema representaron la mayoría con 39\%. Tabla 4.

Tabla 4. Factores intrínsecos, extrínsecos

\begin{tabular}{lcc}
\hline & $\mathbf{f}$ & \% \\
\hline Intrínsecos & 106 & 26 \\
\hline Extrínsecos & 147 & 35 \\
\hline Sistema & 164 & 39 \\
\hline Total & 417 & 100 \\
\hline
\end{tabular}

En relación a los factores intrínsecos, la condición clínica del paciente, en la mayoría de estos no era compleja $110(64 \%)$, y en 63 (36\%) sí era compleja.

Los factores extrínsecos mostraron accesos invasivos de monitorización en 96 (56\%), no invasivo en 40 (23\%), difícil manejo terapéutico 11 (6\%), y no registrado en $26(15 \%)$.

En los factores del sistema referentes al profesional implicado en el incidente, en primera instancia se muestra dentro de los aspectos psicológicos la falta de atención, distracción, preocupación y aburrimiento $47(27 \%)$.

Dentro de los factores de equipo y sociales, la falta de comunicación 39 (23\%). En cuanto a los protocolos, una no adhesión a estos 27 (15\%). Por otro lado, en los factores ligados a la formación y entrenamiento, se encuentran la falta de habilidades 29 (17\%), experiencias 23 (13\%), disposición 21 (12\%), así como falta de supervisión por personal experto 22 (13\%).

Por otro lado, entre los factores ligados a equipamiento y recursos, se presentan la carga de trabajo excesiva $27(16 \%)$ y la presión del tiempo 29 (17\%).

Mediante la prueba exacta de Fisher, se encontraron diferencias significativas, entre ellas: la complejidad de la condición clínica, a mayor complejidad, mayor riesgo de ocurrencia del EA p $<.0001$. A través de la prueba de Chi cuadrada se encontró: fatiga del personal $\mathrm{X}^{2}=9.86 \mathrm{gl}=2 \mathrm{p}<0.05$, falta de comunicación entre el personal $\mathrm{X}^{2}=9.09 \mathrm{gl}=3 \mathrm{p}<0.05$, realización de tareas ajenas $\mathrm{X}^{2}=7.40 \mathrm{gl}=2 \mathrm{p}<0.05$, todo ello relacionado con los factores intrínsecos.

En cuanto a los factores extrínsecos se encontró relación con: EA relacionados al cuidado $\mathrm{X}^{2}=19.81$ $\mathrm{gl}=5 \mathrm{p}<0.001$, falta de habilidades $\mathrm{X}^{2}=9.66 \mathrm{gl}=5 \mathrm{p}<0.05$, y supervisión inadecuada por personal experto $\mathrm{X}^{2}=10.05 \mathrm{gl}=2 \mathrm{p}<0.05$. Y dentro de los factores del sistema: falta de atención, distracción o aburrimiento $\mathrm{X}^{2}=7.98 \mathrm{gl}=2 \mathrm{p}<0.05$.

\section{Discusión}

La edad más frecuente en la que se presentaron los EA fueron en los menores de un año (43\%), a diferencia de un estudio realizado en Utah Colorado, donde se menciona que los eventos presentados en la edad pediátrica, primordialmente son en la adolescencia (13-20 años) $)^{15}$.

Referente a los días de estancia también existe diferencia, reportada por un estudio de la Universidad de Johns Hopkins (Baltimore, EU) ${ }^{16}$, realizado en 27 estados donde mencionan una relación significativa 
asociada al tiempo de estancia (mayor tiempo, mayor riesgo), sin embargo, este estudio mostró que a menor tiempo mayor riesgo, lo cual sucede dentro de los primeros 10 días con un 54\%.

Dentro de los primeros eventos adversos se encuentran: las extubaciones no programadas (16\%), UPP $(14 \%)$, flebitis $(10 \%)$, lesiones $(9 \%)$, quemaduras $(8 \%)$ y medicamentos $(6 \%)$, en similitud con estudios realizados en Brasil ${ }^{13,17}$, donde se percibe que los eventos relacionados con el cuidado, el protagonista principal es el profesional de enfermería, desafortunadamente van en incremento ${ }^{8}$.

Entre los eventos adversos presentados y reportados por quemaduras de diversas causas, los más frecuentes sucedieron por el uso dispositivos (saturómetros, lámparas de calor etc.), esto en concordancia con un estudio realizando en Brasil, donde mencionan escaldaduras y equipamiento como los causantes principales ${ }^{13}$.

Entre los eventos adversos relacionados con los medicamentos, algunos de ellos se presentaron por incumplimiento de los correctos, en semejanza con el estudio de Brasil ${ }^{13}$, dicho evento se presentó por no realizar el proceso de verificación.

Las caídas no tuvieron gran incidencia, no por ello son menos importantes, a diferencia de un estudio realizado en el Seguro Social, donde estas son el principal evento ocurrido ${ }^{18}$.

Se presentaron eventos centinela que ocurrieron en 4\%, que si bien no ocasionaron la muerte del paciente, su vida sí estuvo comprometida y se precisó intervención para mantenerla ${ }^{19}$.

Los eventos adversos son altamente prevenibles, como lo menciona Daniels et al. ${ }^{20}$, hasta en $60 \%$ de los casos, en controversia con este estudio donde los profesionales indicaron que pudieron ser evitables en $93 \%$, lo que demuestra una congruencia con los resultados reportados anteriormente donde se visualizan fallas en los factores del sistema, donde hubo diferencias significativas, principalmente en las actitudes de los profesionales (aburrimiento, falta de disposición, falta de comunicación, entre otros), así como exceso de trabajo pero no por la condición clínica del paciente ${ }^{21}$, sino por situaciones administrativas (llenado de instrumentos, vigilancia de tecnología, etc.). Igualmente, la falta de adhesión a los protocolos, falta de experiencia y habilidades, generan un ambiente propicio para la ocurrencia de dichos eventos.

La supervisión directa del profesional de enfermería encargado del cuidado permite la toma de decisiones basada en la fundamentación del conocimiento científico, amparada en la legislación, así como con garantía de la seguridad y protección del paciente y su familia ${ }^{22}$; ya que en el presente estudio las enfermeras refieren falta de supervisión de personal experto, este es un punto de importancia en la prevención de dichos eventos.

En concordancia con lo que menciona Fernández, el monitoreo de los eventos adversos es un planteo productivo, porque concentra el análisis en hechos que son preocupación tradicional en el ejercicio profesional. Se trata de una evaluación de la calidad focalizada en los problemas evitables, cada institución considerará aquellos problemas que le interesa estudiar y controlar. Luego de ello se instalará una serie de actividades tendientes a evaluar primero y mejorar después la situación encontrada ${ }^{23}$. Actualmente, el énfasis se encuentra en el análisis de las causas y los cambios que se pueden introducir en los recursos, procedimientos o conductas, según corresponda, para minimizar los daños y costos que esto conlleva.

\section{Conclusiones}

Las áreas donde se reportó mayor ocurrencia de eventos fue en las críticas (neonatos, UTI, UTI cardiovascular, urgencias) con un $60 \%$ y hospitalización $40 \%$, es indicativo que sea en sitios de alta complejidad, con manejo importante de tecnología, dispositivos y numerosos instrumentos de valoración, donde es fácil que el personal distraiga su atención de la clínica del paciente, desviándola al entorno, por lo cual es probable que esto influya en el incremento de la ocurrencia.

En cuanto a la clasificación de gravedad, el 53\% presentó un Grado III de la escala TISS, donde se requiere de una vigilancia intensiva y la relación enfermera / paciente es 1:2. A pesar de que se presentó 
este grado, la condición clínica no era compleja, lo cual refleja que el evento se puede presentar debido a factores intervinientes como distracción, preocupación, sobrecarga o aburrimiento.

El turno predominante fue el nocturno con $46 \%$, esto es sugerente de agotamiento del personal que conlleva un factor de riesgo no sólo para el profesional, sino en la manera de brindar el cuidado.

Aproximadamente, la mitad de estos eventos es informado a la familia (49\%), no obstante, el cuidador primario y el paciente tienen derecho por una parte, a saber que ha pasado y por otra, a reclamar ayuda, apoyo y reparación del daño causado. La comunicación es un factor de competencia diaria de los profesionales de la salud, ya que establece una interacción con el niño, el cuidador y su familia, lo cual es favorable en el proceso terapéutico. De esta manera, permite un cuidado más humanizado y aclarar dudas sobre los procedimientos que se realizan. El proceso de comunicación efectiva, cuando se adopta de forma interdisciplinaria, favorece una atención de salud segura.

Los pacientes suelen responder de forma positiva al proceso de información abierta y honesta, por lo tanto mejora la relación asistencial y la confianza en el sistema sanitario.

El manejo de las sondas a pesar de parecer procedimientos sencillos, conllevan graves riesgos para el paciente que interfirien en la continuidad de la atención, por lo que una extubación no programada trae grandes riesgos, como lo es un paro cardiorespiratorio que compromete la vida de los pacientes.

Las úlceras por presión se caracterizan por ser lesiones altamente prevenibles, cuando se realiza un adecuado manejo como la movilización frecuente, inspección y protección de la piel, así como una monitorización continúa, ya que no sólo representa incremento de la estancia y costos hospitalarios, sino también sufrimiento físico y emocional de los pacientes.

Los eventos adversos relacionados con la flebitis se deben a diversos factores como: administración de soluciones y medicamentos que sobrepasan el nivel de acidez o alcalinidad de la sangre, o como osmolaridad aumentada por la dosis, cantidad y tipo de diluyente, aspectos relacionados con la infusión (tiempo, velocidad, etc.), además de tiempo prolongado de colocación del catéter

El manejo del paciente que se encuentra en áreas críticas, suele ser multi-invasivo y con monitorización continua, esto determina que el tiempo de colocación de los dispositivos sea prolongado y genere la aparición de lesiones que complican su estado.

En cuanto a las IAAS y a las pruebas diagnósticas, no se reportó ningún evento relacionado, lo cual no significa que no se hayan presentado, simple y sencillamente no estamos acostumbrados a reportarlos como eventos adversos.

Entre los factores relacionados a la seguridad se encuentran; el ambiente de trabajo, cuestiones individuales y colectivas del equipo de salud, así como aspectos institucionales.

Dentro de la fuerza laboral se encuentran las enfermeras, quienes constituyen un grupo básico y mayoritario en las instituciones de salud, por lo tanto se convierten en los principales actores del sector salud, ya que participan activamente en la entrega de cuidados directos e indirectos a personas, familias y a la comunidad, por lo cual es entendible que esté involucrado en el $90 \%$ de los eventos ocurridos.

Las condiciones de trabajo y el tipo de contratación como la provisionalidad representan pérdida de la estabilidad laboral, desprotección de la seguridad social y de prestaciones sociales. La amplia gama de funciones que debe cumplir el profesional de enfermería: distribución de trabajo al personal; disposición del personal en los distintos días, turnos y servicios; consecución de ropas, materiales, medicamentos y aparatos; puesta al día de información en kárdex y tarjetas de medicamentos, atención a las demandas de los familiares de los pacientes, coordinación de los servicios de diagnóstico y tratamiento, atención de los pacientes, llenado de numerosos instrumentos de valoración, etc., diluye el perfil de su rol y lo dispersan en un sinnúmero de tareas que se apartan del objetivo mismo de la enfermería, lo que conlleva a la aparición de los eventos adversos.

Se sabe que los eventos adversos van a continuar sucediendo, sin embargo al implementar acciones seguras dirigidas a la protección del paciente, existe una tendencia a la disminución, para con ello dar realce a la calidad y seguridad en los cuidados que brinda el profesional de enfermería. Evidentemente, 
es necesario realizar una mayor concientización de los profesionales en la identificación de la profesión, de la misma manera incorporar un cuidado humanizado, y de los gestores para la reformulación de las políticas institucionales e implementación de programas, que apoyen las medidas de seguridad y protección a los usuarios en el ambiente hospitalario.

\section{Responsabilidades éticas}

Protección de personas y animales. Los autores declaran que para esta investigación no se han realizado experimentos en personas o animales.

Confidencialidad de los datos. Los autores declaran que en este artículo no aparecen datos de pacientes. Financiamiento. Trabajo derivado del proyecto multicéntrico con apoyo de PAPIIT-UNAM IN304414 Conflicto de intereses. Los autores declaran no tener conflicto de intereses.

\section{Referencias}

1. Organización Mundial de la Salud. Alianza mundial para la seguridad del paciente: la investigación en seguridad del paciente, mayor conocimiento para la atención más segura. Francia: OMS; 2008. [Consultado octubre 20 2017]. Disponible en: http://bit.ly/2q9P5G4

2. Aranaz JM, Aibar C, Vitaller J, Ruiz P. Estudio Nacional sobre los efectos adversos ligados a la Hospitalización. ENEAS 2005. España: Ministerio de Sanidad y Consumo; 2006.

3. Rodríguez-Suárez J, Santacruz-Varela J, Fajardo-Dolci G, Hernández-Torres F. Sistemas de notificación y registro de incidentes en México: aprendizajes. Revista CONAMED. 2012; 17(2): 82-6.

4. Wilson RM, Michel P, Olsen S, Gibberd RW, Vincent C, El-Assady R, et al. Patient safety in developing countries: Restrospective estimation of scale and nature of harm to patients in hospital. BMJ. 2002; 344:1-14. https://doi.org/10.1136/bmj.e832

5. Ramírez-Elizondo N, Paravic-Klijn T, Valenzuela-Suazo S. Riesgo de los turnos nocturnos en la salud integral del profesional de enfermería. Index Enferm 2013; 22(3): 152-5.

http://dx.doi.org/10.4321/S1132-12962013000200008

6. Organización Mundial de la Salud. 10 datos sobre seguridad del paciente. Ginebra: OMS; 2018. [Consultado marzo 2 2018]. Disponible en: https://bit.ly/2QIbExG

7. Delgado MC, Fernández M, Anglés I, Coll R, Palencia E, Catalán RM, et al. Información de eventos adversos a pacientes y familiares. Trauma Fund MAPFRE 2013; 24(4): 239-48. [Consultado marzo 1 2016]. Disponible en: https://bit.ly/2MCSNE9

8. Organización Mundial de la Salud. Gasto en salud. Ginebra: OMS; 2018. [Consultado 2 marzo 2018]. Disponible en https://bit.ly/2Wy5ip9

9. Rodríguez Rey YL. Causas de ocurrencia de evento adverso relacionado con la atención de enfermería. [Tesis de grado]. Colombia: Universidad Nacional de Colombia. Facultad de Enfermería. 2014. p. 40

10. Riquelme-Hernández G, Ourcilleón-Antognoni AA. Descripción de eventos adversos en un hospital pediátrico de la ciudad de Santiago, Chile. Enferm. glob. 2013; 12(29): 262-73. [Consultado marzo 2 2018]. Disponible en: https://bit.ly/2HH2ylM

11. Martínez-Reyes SR. Eventos Adversos en el cuidado de Enfermería brindado a niños hospitalizados. REVISALUD Unisucre 2014; 2(1): 66 - 9. [Consultado marzo 02 2016]. Disponible en: https:// bit.ly/2FWzdC3

12. Zárate-Grajales R, Olvera-Arreola S, Hernández-Cantoral A, Hernández-Corral S, Sánchez-Angeles S, Valdez-Labastida R, et al. Factores relacionados con eventos adversos reportados por enfermería en unidades de cuidados intensivos. Proyecto multicéntrico. Enferm univ. 2015; 12(2): 63-72. http://dx.doi.org/10.1016/j.reu.2015.03.003

13. Silva T, Wegner W, Pedro ENR. Segurança da criança hospitalizada na UTI: compreendendo os eventos adversos sob a ótica do acompanhante. Rev. Eletr. Enf 2012; 14(2): 337-44. Disponible en: http://dx.doi.org/10.5216/ree.v14i2.12977 
14. Zárate-Grajales RA, Salcedo-Álvarez RA, Olvera-Arreola SS, Hernández-Cantoral S, BarrientosSánchez J, Pérez López MT, et al. Eventos adversos en pacientes hospitalizados reportados por enfermería: un estudio multicéntrico en México. Enferm univ. 2017; 14 (4): 277-85. https://doi.org/10.1016/j.reu.2017.08.005

15. Woods D, Thomas E, Holl J, Altman S, Brennan T. Adverse events and preventable adverse events in children. Pediatrics 2005; 115(1): 155-60. https://doi.org/10.1542/peds.2004-0410

16. Miller MR, Zhan C. Pediatric patient safety in hospitals: a national picture in 2000. Pediatrics 2004; 113(6): 1741-6. [Consultado febrero 29 2016]. Disponible en: https://bit.ly/2BbpfbX

17. Moreira IA, Bezerra ALQ, Paranaguá TTB, Silva AEBC, Filho FMA. Conhecimento dos profissionais de saúde sobre eventos adversos em unidade de terapia intensiv. Rev enferm UERJ. 2015; 23 (4): 461-7 http://dx.doi.org/10.12957/reuerj.2015.5158

18. Ornelas-Aguirre JM, Arriaga-Dávila JJ, Domínguez-Serrano MI, Guzmán-Bihouet BF, NavarreteNavarro S. Eventos adversos en pacientes de un hospital pediátrico. Rev. Med. Inst. Mex. Seguro Soc. 2013; 51(6): 680-7. [Consultado febrero 25 2016]. Disponible en: https://bit.ly/2FZPwyd

19. The Joint Commission. Sentinel Event Policy and Procedures (Topic Library Item). Washington DC: Joint Commission 2016 [Consultado marzo 03 2016). Disponible en: https://bit.ly/2RsIkMm

20. Daniels J, Hunc K, Cochrane D, Carr R, Shaw N, Ansermino J, et al. Identification by families of pediatric adverse events and near misses overlooked by health care providers. CMAJ: 2012; 184(1): 29-34. http://dx.doi.org/10.1503/cmaj.110393

21. García-de Lorenzo A. Intervención terapéutica. En: Scores pronósticos y criterios diagnósticos. $2^{\mathrm{da}}$ ed. España: Ergón S.A.; 2006.

22. Modelski-Schatkoski A, Wegner W, Algeri S, Rubim-Pedro EN. Safety and protection for hospitalized children: literature review. Rev Latino-am Enfermagem 2009; 17 (3): 410-6. http://dx.doi.org/10.1590/S0104-11692009000300020

23. Fernández-Busso N. Los eventos adversos y la calidad de atención: Estrategias para mejorar la seguridad de los pacientes pediátricos. Arch. argent. pediatr. 2004; 102(5): 402-10. [Consultado agosto 23 2018]. Disponible en: https://bit.ly/2BcCkBP 